\title{
Evaluación de factores de riesgo foniátricos y generales de disfonía en tiroidectomías*
}

\author{
Dr. IGNACIO GOÑI E. ${ }^{1}$, Int. CLAUDIO KRSTULOVIC R. ${ }^{2}$, Dra. ALEJANDRA QUINTAS W. ${ }^{1}$, \\ AUGUSTO LEÓN R. ${ }^{1}$, HERNÁN GONZÁLEZ D. ${ }^{1}$, MANÉ LEÓN M. ${ }^{3}$, \\ NICOLÁS DROPPELMANN M. ${ }^{1}$, EU CATALINA RUIZ A. ${ }^{1}$ \\ 1 Departamento de Cirugía Oncológica y Maxilofacial, División de Cirugía. \\ 2 Interno Escuela de Medicina. \\ 3 Departamento de Otorrinolaringología. Fonoaudiología. \\ Pontificia Universidad Católica de Chile. \\ Santiago, Chile.
}

Abstract

\section{Thyroidectomy dysphonia}

Background: Dysphonia is one of the most important and frecuent complication in thyroidectomies. It incidence is between 0 and $20 \%$. The frecuency of this complication depends on factors like the preoperatory diagnosis, goitre volume, surgeon experience and other general factors that theoretically can affect it incidence. Aim: To evaluate a group of preoperatory risk factors usually not considerated that may have relevance in postoperatory dysphonia in thyroidectomies. Patients and Method: Survey and medical records of 56 patients operated between may and july 2009. Results: None of the risk factors included in this study increase de incidence of posthiroidectomy dysphonia. Conclusions: It seems that these theorical risk factors don't have a great influence in the incidence of dysphonia after a thyroidectomy. It can be eventually useful to submit these patients to a direct vocal cord evaluation before surgery, to obtain their real risk.

Key words: Thyroidectomy, dysphonia.

\section{Resumen}

Introducción: La disfonía es una de las complicaciones en tiroidectomías más importante y frecuente. Su incidencia varía entre 0 y 20\%. Esta incidencia depende de factores como el tipo de diagnóstico preoperatorio, el volumen del bocio, la experiencia del cirujano y otros factores generales que pueden teóricamente influir. Objetivo: Evaluar un grupo no habitualmente considerado de factores de riesgo de disfonía en tiroidectomías. Pacientes y Métodos: Estudio prospectivo con análisis de fichas clínicas y realización de una encuesta preoperatoria en 56 pacientes consecutivos. Resultados: En este estudio no fue demostrado estadísticamente un mayor riesgo de disfonía en pacientes con los factores de riesgos planteados. Conclusiones: Aunque los factores estudiados en esta muestra pueden teóricamente aumentar la incidencia de disfonía postiroidectomía, parecen no ser los más influyentes. Podría ser de utilidad realizar una evaluación dirigida otorrinolaringológica en estos pacientes para objetivar su real riesgo de disfonía postoperatoria.

Palabras clave: Tiroidectomía, disfonía.

*Recibido el 11 de junio de 2010 y aceptado para publicación el 31 de agosto de 2010.

Correspondencia: Dr. Ignacio Goñi E. Marcoleta 350, Santiago, Chile igoni@med.puc.cl 


\section{Introducción}

Siendo la tiroidectomía una intervención en general reglada y con baja frecuencia de complicaciones serias y permanentes, éstas son de connotación muy relevante para el paciente y el manejo postoperatorio. Entre ellas destaca la disfonía, que siendo generalmente transitoria, origina ansiedad en el paciente, retrasa la reincorporación laboral y puede ser motivo de incidentes médico-legales. Junto al hipoparatiroidismo postoperatorio y al hematoma brusco del cuello, constituyen las tres complicaciones más características y relevantes de esta cirugía ${ }^{1,2}$.

La incidencia de disfonía en tiroidectomías varía entre 0 y $20 \%{ }^{3-5}$, dependiendo de los criterios de definición y de varios factores, siendo los más importantes, en análisis multivariados, la extensión de la resección y la cirugía del bocio recurrente ${ }^{1,3}$. En esta cifra también incide el diagnóstico preoperatorio (más frecuente en tiroidectomías por hipertiroidismo o bocio de gran tamaño) $)^{3}$, la experiencia del equipo quirúrgico y , eventualmente, otros factores que son de aspecto general como el hábito tabáquico, la obesidad, la patología cordal previa, hábito de voz, las enfermedades reactivas y obstructivas bronquiales, el reflujo gastroesofágico, etc 6 .

De esta forma, se han considerado una serie de factores que podrían aumentar el riesgo de esta complicación. Conocer estos elementos permitiría explicar al paciente su eventual mayor riesgo de disfonía, independiente de los factores intraoperatorios considerados habitualmente.

\section{Material y Método}

Entre mayo y julio de 2009 se aplicó una encuesta clínica preoperatoria (al momento del ingreso hospitalario) a 56 pacientes consecutivos con indicación de tiroidectomía, que incluía los siguientes elementos a evaluar:

- datos personales

- tipo de operación

- y las siguientes preguntas en la encuesta:

- ¿nota cambios en la voz?

- ¿se pone ronco (a) con facilidad?

- ¿se esfuerza al hablar?

- ¿̇amanece ronco (a)?

- ¿Presenta problemas en la voz que limiten su vida personal, laboral o social?

- ¿ ¿la gente le pregunta si tiene problemas en la voz?

- ¿le cuesta escuchar?

- ¿ha consultado alguna vez a un otorrinolaringólogo o fonoaudiólogo por su voz?
- y las siguientes patologías y hábitos:

- canta

- fuma, cuánto

- actividad o trabajo

- peso/talla

- reflujo gastroesofágico

- hipoacusia

- tos reciente o actual

- asma, EPOC, rinitis

- enfermedad mesenquimática

- hipotiroidismo reciente

- patología psiquiátrica

- radioterapia regional

Estos factores fueron agrupados en los que dependen de fenómenos foniátricos propiamente tales, que se refieren al uso de la voz, y los que derivan de patologías concomitantes y hábitos que pueden incidir en la frecuencia de disfonía.

Al día siguiente de la operación, se registró la presencia o ausencia de disfonía. Igualmente se evaluó telefónicamente o en los controles postoperatorios la persistencia o desaparición de ella. Además, fueron revisadas las fichas clínicas de estos pacientes para registrar la histología definitiva de su patología tiroídea.

Para el análisis de los resultados, los pacientes fueron divididos en dos grupos, siendo uno el de los pacientes sin disfonía (grupo 1) y el otro, de los pacientes con disfonía postoperatoria (grupo 2). Se utilizó un estudio estadístico descriptivo, el test $\mathrm{T}$ de Student y el test de $\chi^{2}$, para el análisis de los resultados.

La técnica quirúrgica utilizada es estándar y se realiza bajo anestesia general con intubación orotraqueal, incisión transversa y abordaje de la glándula tiroides. Se realizaron ligaduras a los vasos principales, con identificación rutinaria de ambos nervios recurrentes laríngeos y de las glándulas paratiroides. La hemostasia de vasos menores fue prolija y realizada con electrocoagulador bipolar. En todos se realizó tiroidectomía total.

\section{Resultados}

Las características de los pacientes en ambos grupos se detalla en la Tabla 1.

La incidencia de disfonía fue de 39\% (grupo 2). Ésta varió en magnitud subjetiva y fue registrada como presente si era señalada por el o la paciente o era clínicamente evidente, ya sea en el postoperatorio inmediato (17 pacientes) o posteriormente (5 pacientes). No se hizo diferencia entre disfonía transitoria o permanente, ni del tipo (disfonía constante, voz bitonal, dificultad con tonos altos, etc). 
EVALUACIÓN DE FACTORES DE RIESGO FONIÁTRICOS Y GENERALES DE DISFONÍA EN TIROIDECTOMÍAS

Tabla 1. Características de los grupos

\begin{tabular}{lcc}
\hline Característica & $\begin{array}{c}\text { Grupo 1 (n/\%) } \\
\text { Sin disfonía }\end{array}$ & $\begin{array}{c}\text { Grupo 2 ( n/\%) } \\
\text { Con disfonía }\end{array}$ \\
\hline n de pacientes & $34 / 61$ & $22 / 39$ \\
Sexo & & \\
$\quad$ masculino & $5 / 14$ & $0 / 0$ \\
$\quad$ femenino & $29 / 86$ & $22 / 100$ \\
Rango de edad & $25-68$ & $23-68$ \\
Promedio de edad & 48,8 & 43,2 \\
\hline
\end{tabular}

Tabla 3. Características y uso de la voz en preoperatorio (Sólo pacientes con respuesta afirmativa)

\begin{tabular}{|c|c|c|c|}
\hline Característica & $\begin{array}{c}\text { Grupo } 1 \\
(\mathbf{n} / \%)\end{array}$ & $\begin{array}{c}\text { Grupo } 2 \\
\text { (n/\%) }\end{array}$ & $\mathbf{p}$ \\
\hline Cambios en la voz & $7 / 21$ & $3 / 14$ & 0,358 \\
\hline Ronquera reciente & $14 / 41$ & $8 / 36$ & 0,921 \\
\hline Esfuerzo al hablar & $4 / 12$ & $0 / 0$ & 0,095 \\
\hline Amanecer ronco & $13 / 38$ & $6 / 27$ & 0,488 \\
\hline $\begin{array}{l}\text { Problemas de voz que } \\
\text { limitan vida personal o } \\
\text { laboral }\end{array}$ & $2 / 6$ & $0 / 0$ & 0,247 \\
\hline $\begin{array}{l}\text { Le preguntan si tiene } \\
\text { problemas en la voz }\end{array}$ & $6 / 17$ & $2 / 9$ & 0,358 \\
\hline Le cuesta escuchar & $7 / 20$ & $2 / 9$ & 0,247 \\
\hline $\begin{array}{l}\text { Consulta a OTR o FA } \\
\text { por su voz }\end{array}$ & $3 / 9$ & $0 / 0$ & 0,359 \\
\hline
\end{tabular}

OTR: Otorrino. FA: Fonoaudiólogo.

No hubo evaluación nasofibroscópica de rutina en estos pacientes.

La mayoría de los pacientes fueron mujeres. Los 5 hombres operados no tuvieron disfonía.

Los rangos de edad y promedios no fueron distintos estadísticamente ( $\mathrm{T}$ de Student, $\mathrm{p}=0,090$ ).

El diagnóstico histológico definitivo y su relación con disfonía se muestran en la Tabla 2.

El diagnóstico histológico definitivo maligno estuvo presente en $59 \%$ de los pacientes del grupo 1 y en $64 \%$ del grupo 2 (con disfonía). Estas cifras no fueron estadísticamente diferentes $(\mathrm{p}=0,719)$. No hubo relación alguna entre histología definitiva y presencia de disfonía.

En la Tabla 3 se detalla el análisis de la encuesta practicada a los pacientes antes de su cirugía en relación a características y uso de la voz.

Ninguno de estos posibles factores de riesgos relacionados con alguna alteración o uso de la voz
Tabla 2. Incidencia de disfonía según la histología definitiva

\begin{tabular}{lccc}
\hline Patología & $\begin{array}{c}\text { Grupo 1 } \\
(\mathbf{n} / \%)\end{array}$ & $\begin{array}{c}\text { Grupo 2 } \\
\text { (n/\%) }\end{array}$ & p \\
\hline Patología maligna & $20 / 59$ & $14 / 64$ & 0,719 \\
Patología benigna & $14 / 41$ & $8 / 36$ & 0,719 \\
Total & 34 & 22 & \\
\hline
\end{tabular}

Tabla 4. Patología concomitante y hábitos preoperatorios (basado en antecedentes documentados o subjetivos)

\begin{tabular}{lccc}
\hline Antecedente & $\begin{array}{c}\text { Grupo 1 } \\
\text { (n/\%) }\end{array}$ & $\begin{array}{c}\text { Grupo 2 } \\
\text { (n/\%)-p }\end{array}$ & p \\
\hline Canta & $5 / 15$ & $4 / 18$ & 0,729 \\
Fuma & $7 / 21$ & $9 / 40$ & 0,665 \\
Sobrepeso* & $18 / 53$ & $15 / 68$ & 0,312 \\
Reflujo gastroesofágico & $7 / 21$ & $6 / 27$ & 0,563 \\
Hipoacusia & $2 / 6$ & $1 / 5$ & 0,544 \\
Tos reciente o actual & $8 / 23$ & $8 / 36$ & 0,299 \\
Asma, EPOC, rinitis & $1 / 3$ & $0 / 0$ & 0,410 \\
Enf. mesenquimática & $0 / 0$ & $1 / 5$ & 0,210 \\
Hipotiroidismo & $2 / 6$ & $4 / 18$ & 0,146 \\
Patología psiquiátrica & $8 / 24$ & $5 / 23$ & 0,945 \\
Radioterapia regional & $0 / 0$ & $0 / 0$ & \\
\hline
\end{tabular}

Según IMC (índice de masa corporal). EPOC: Enfermedad pulmonar obstructiva crónica.

aumentaron estadísticamente el riesgo de presentar disfonía.

Los resultados de la evaluación de patología concomitante y hábitos se detallan en la Tabla 4.

Según los resultados mostrados en esta tabla, no hubo diferencia estadística en la incidencia de disfonía en los pacientes con estos antecedentes.

\section{Discusión}

La tiroidectomía corresponde actualmente a una de las cirugías más frecuentes de los centros asistenciales en el país. En el Hospital Clínico de la Pontificia Universidad Católica de Chile, esta cirugía ocupa el cuarto lugar de frecuencia con alrededor de $300 \mathrm{a}$ 400 casos anuales (datos internos).

En este estudio, que analiza la importancia de ciertos factores habitualmente no considerados en 
la evaluación de los pacientes con indicación de cirugía tiroídea en relación al riesgo de disfonía, se estableció un análisis prospectivo de 56 pacientes consecutivos, utilizando los datos clínicos y una encuesta, detallada más arriba.

Los dos grupos a comparar, esto es los que no presentaron disfonía postoperatoria (grupo 1) y los que sí la presentaron (grupo 2), tienen una distribución etaria y de género similares y concordantes con diferentes series publicadas de cirugía tiroídea ${ }^{2,8}$.

La incidencia de disfonía en esta serie de 56 pacientes fue de $39 \%$, que es mayor a lo reportado habitualmente (0 a 20\%1,3,4,8). Esto podría explicarse dado que se consideró como disfonía cualquier alteración de la voz en el postoperatorio o en el seguimiento inicial, incluyendo la opinión, subjetiva por cierto, del o de la paciente. No se realizó en esta etapa del estudio una evaluación objetiva con procedimientos de apreciación fonoaudiológica ni endoscópica. La incidencia de disfonía permanente en nuestro grupo corresponde a la de la literatura ${ }^{1}$. Para el análisis de la incidencia de disfonía en estos casos, se requiere de definiciones que permitan una evaluación más precisa.

El grupo con disfonía (grupo 2) está compuesto porcentualmente por más tiroidectomías por patología tumoral maligna que benigna, lo que está de acuerdo con la literatura. Sin embargo, en nuestra serie no se evidenció diferencia estadística. El cáncer de tiroides condicionaría una cirugía eventualmente más compleja, dependiendo del volumen tumoral, e invade el nervio recurrente laríngeo más frecuentemente que otras estructuras ${ }^{4}$. En nuestro centro $^{8}$, un tercio de los pacientes tienen diagnóstico de microcarcinoma diferenciado de tiroides, explicado por pesquisa y estudio precoz de las lesiones nodulares tiroídeas, lo que disminuye el riesgo de compromiso extraglandular y, por lo tanto, de la invasión de estructuras vecinas a la glándula como los nervios recurrentes laríngeos. Algunos autores recomiendan la realización de estudio endoscópico rutinario de las cuerdas vocales preoperatoriamente cuando se establece el diagnóstico de cáncer de tiroides ${ }^{7}$.

En la Tabla 3 se registran los resultados de los elementos evaluados en la encuesta sobre uso de la voz. Desde el punto de vista fonoaudiológico, existen elementos clínicos que permiten sugerir la presencia de alguna patología cordal o malos hábitos de voz en estos pacientes, que incidirían en una cierta susceptibilidad a que la cirugía de tiroides origine disfonía postoperatoria, agravando una condición preexistente $^{9,10}$. Contrario a esta hipótesis, nuestros resultados no mostraron mayor riesgo de disfonía en pacientes con algunos de estos elementos. Como conducta recomendada ante la sospecha de este tipo de patologías, se indicaría una evaluación laringoscópica indirecta o nasofibroscópica preoperatoria.

Igualmente ocurrió que no se demostró en este estudio una mayor incidencia de disfonía en pacientes con hábitos de esfuerzo de la voz (canto), tabáquico o patologías relacionadas con patología cordal secundaria.

\section{Conclusión}

La incidencia de disfonía postiroidectomía varía importantemente según la forma de definirla y la acuciosidad diagnóstica.

Los factores de riesgo relacionados con el uso de la voz y la comorbilidad y hábitos que fueron establecidos en este estudio, no influyeron en la incidencia de disfonía postoperatoria, de acuerdo a nuestros resultados. En estos pacientes, un estudio invasivo (nasofibroscopia) dirigido de las cuerdas vocales podría ser de utilidad en establecer un riesgo más real de disfonía, así como establecer elementos más precisos en la evaluación clínica inicial ante estos antecedentes.

\section{Referencias}

1. O, Machens A, Sekulla C, Ukkat J, Lippert H, Gastinger I, Dralle H. Multivariate analysis of risk factors for postoperative complications in benign goiter surgery: prospective multicenter study in Germany. World J Surg. 2000;24:1335-41.

2. Claure R. Morbilidad en tiroidectomías. Rev Chil Cir. 1987;3:187-94.

3. Dralle H, Sekulla C, Haerting J, Timmermann W, Neumann HJ, Kruse E, et al. Risk factors of paralysis and functional outcome after recurrent laryngeal nerve monitoring in thyroid surgery. Surgery 2004;136:131022.

4. Benavides MP. Disfonía. Guías clínicas, Lugo, España 2007.

5. Petter V, Barros de Oliveira PA, Fischer PD. Relación entre disfonía referida y potenciales factores de riesgo en el trabajo de profesores de la enseñanza fundamental, Porto Alegre - RS. Salud de los Trabajadores 2006;14:5-12.

6. Frye JW, Vaezi MF. Extraesophageal GERD. Gastroenterol Clin North Am. 2008;37:845-58.

7. Randolph G, Kamani D. The importance of preoperative laryngoscopy in patients undergoing thyroidectomy: voice, vocal cord, function and the preoperative detection of invasive thyroid malignancy. Surgery 2006;139:357-64.

8. Fardella C, Jiménez M, González H, León A, Goñi I, Cruz F, y cols. Características de presentación del microcarcinoma papilar de tiroides. Experiencia retros- 
pectiva de los últmos 12 años. Rev Med Chile 2005; 133:1305-10.

9. Eisenbeis JF, Fuller DP. Voice disorders: abuse, misuse and functional problems. Mo Med. 2008;105:240-3.

10. Franco RA, Andrus JG. Common diagnoses and treatments in professional voice users. Otolaryngol Clin
North Am. 2007;40:1025-61.

11. Ozbas S, Kocak S, Aydintug S, Cakmak A. Demirkiran MA, Wishart G. Comparision of the complications of subtotal, near total and total thyroidectomy in the surgical manegement of multinodular goitre. Endocrine Journal 2005;52:199-205. 